\title{
Distribución de fuerzas en medios granulares no cohesivos: Observaciones experimentales y computacionales.
}

\section{Force distribution in none cohesive granular media: Experimental and Computational Observations}

\author{
Watson L. Vargas ${ }^{a}$, Juan Carlos Murcia ${ }^{b}$, \\ Facultad de Ingeniería \\ Universidad Militar Nueva Granada \\ Bogotá, D.C., Colombia
}

\section{Resumen}

La comprensión de las propiedades mecánicas de los medios granulares es importante en aplicaciones de Ingeniería civil, Ingeniería química, ciencias geofísicas al igual que en otras áreas del conocimiento. Es bien sabido que las fuerzas dentro de un medio granular se distribuyen siguiendo una red altamente no-lineal de partículas bajo esfuerzo. Las fuerzas por arriba del promedio se concentran en una red de "cadenas de esfuerzo" que soportan la gran mayoría de la carga, mientras que existen partículas dentro del lecho que se encuentran prácticamente aisladas de sus vecinas y que no soportan carga alguna. Dado que las fuerzas inter-partícula y su distribución determinan las propiedades macroscópicas de un sistema granular, dicho comportamiento tiene consecuencias muy importantes en fenómenos de transporte tales como conducción de calor, propagación de sonido y conducción eléctrica. En este trabajo se presentan resultados de una investigación tanto experimental como computacional sobre el comportamiento de la distribución de esfuerzos en un medio granular estático. Los resultados expuestos proveen conocimientos nuevos sobre la física de medios granulares y complementan observaciones previas.

Palabras clave: Medios granulares, cadenas de esfuerzo, Dinámica de Elementos Discretos, DEM

\section{Abstract}

\footnotetext{
${ }^{a}$ Profesor Asociado, Facultad de Ingeniería (UMNG). Ing. Químico, MSc., Ph.D Autor corresponsal: wvargas@etb.net.co

${ }^{\mathrm{b}}$ Ing. Civil, Estudiante de Maestría Universidad de los Andes
} 
Understanding the behavior of mechanical properties within granular media is essential in several applications of civil and chemical engineering, geophysical sciences as well as in many other areas of science. It is well established that in non-cohesive granular media, the transmission of force occurs exclusively via inter particle contacts, which follow preferred paths, the so-called "stress chains" or "force chains" network. Because the inter-particle forces and its distribution determine the bulk properties of a granular system, this behavior has very important consequences in transport phenomena as well as in constitutive modeling. In this work we present results, both experimental and computational, on the behavior of the force distribution in static granular materials. These results provide new insight into the physics of granular materials and complement previous findings.

Keywords: Granular media, stress chains, Discrete Element Method, DEM

\section{Introducción}

Está claramente establecido que dentro de un medio granular no cohesivo, la transmisión de fuerzas ocurre exclusivamente vía contacto entre partículas y que dichas fuerzas se distribuyen siguiendo una red altamente no lineal de partículas, las denominadas "cadenas de esfuerzo". Un ejemplo de dichas cadenas de esfuerzo se ilustra en la Figura 1. Dado que la fuerza inter-partícula y su distribución determinan las propiedades macroscópicas de un sistema granular, su comportamiento tiene consecuencias muy importantes en fenómenos de transporte tales como conducción de calor ${ }^{1}$, propagación de sonido $^{2}$ y conducción eléctrica ${ }^{3}$.
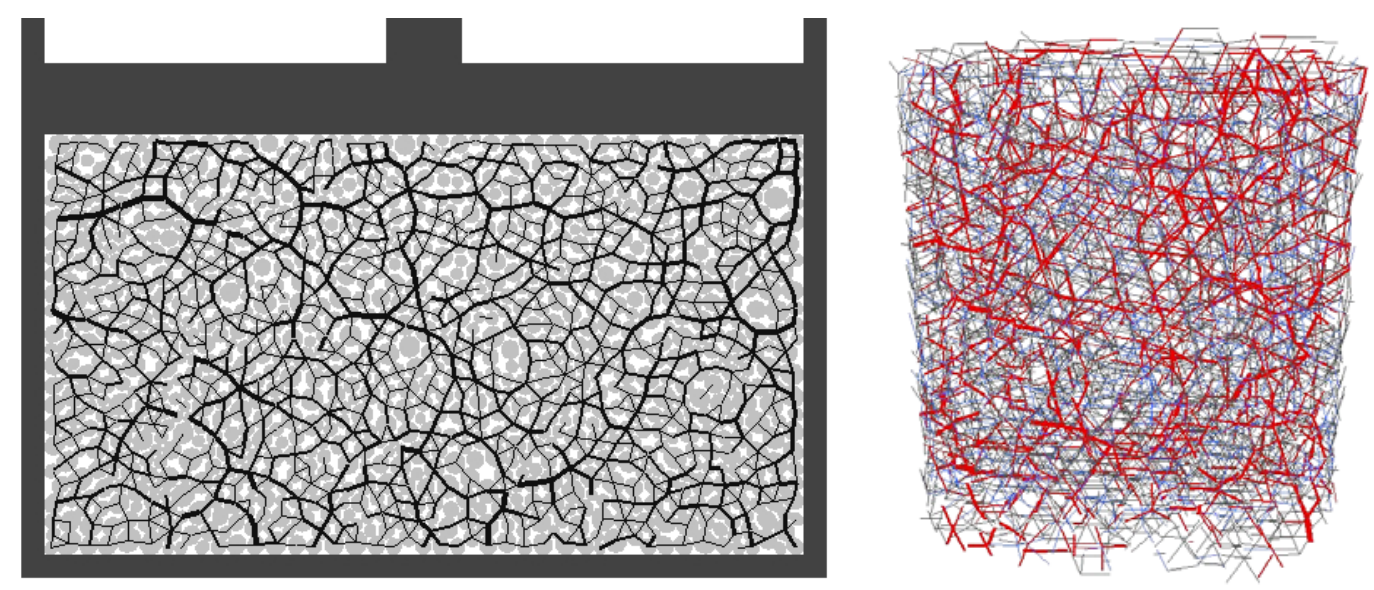

Figura 1. Red de fuerzas normales en un lecho bidimensional y tridimensional bajo carga. Las líneas de contacto ínter-centros más anchas indican esfuerzos mayores. Notar que existen partículas dentro del lecho que no soportan carga alguna. 
Una forma común de analizar la distribución de esfuerzos en medios granulares es la de determinar la función de distribución de probabilidad $P(f)$ de las fuerzas normales entre partículas vecinas, donde $f=F /\langle F\rangle$, y $\langle F\rangle$ indica el valor promedio. La distribución de esfuerzos dentro de un medio granular es un proceso complejo que depende de las propiedades del material, la carga externa aplicada así como de la gravedad, el ordenamiento del empaquetamiento, fricción, geometría del contenedor y de la forma como se construya el sistema. Observaciones tanto experimentales ${ }^{4}$ como computacionales ${ }^{5}$ indican que la función de distribución de probabilidad $P(f)$ presenta varias características comunes que incluyen un decaimiento exponencial para fuerzas por arriba del promedio i.e., $f \geq 1$ y un pico o plateau alrededor de la fuerza promedio $f \approx 1$. El comportamiento en el límite $f \rightarrow 0$ aún permanece en controversia, sin embargo la gran mayoría de datos experimentales y computacionales indican que $P(f)$ se aproxima a un valor finito. Las características observadas son las mismas tanto en dos como en tres dimensiones. Formas funcionales tanto empíricas ${ }^{4,6}$ como teóricas $^{7}$ han sido propuestas para modelar la distribución de tales fuerzas. Dichos modelos capturan la mayoría de las características mencionadas anteriormente y siguen trayectorias similares dentro de ciertos límites.

Muchas preguntas acerca de la distribución de esfuerzos en un medio granular aún permanecen sin respuesta. Aún para sistemas particulados mono-dispersos dicho comportamiento no se ha comprendido enteramente. El comportamiento de la distribución de esfuerzos así como algunas otras características propias de la micro-estructura granular, que no son fácilmente medibles experimentalmente, son el foco del presente artículo. En este estudio se presentan resultados tanto experimentales como computacionales de la compresión estática de mezclas binarias de partículas blandas y duras, distribuidas espacialmente de manera aleatoria y/o estructurada.

El artículo está organizado como sigue. La Sección II presenta el método numérico utilizado en el estudio. En la Sección III se presenta resultados sobre la propagación tridimensional de esfuerzos en mezclas aleatorias binarias de partículas. Una comparación con modelos existentes y observaciones experimentales también se examinan en esta sección. Finalmente en la Sección $\mathrm{IV}$, se proveen algunas conclusiones y perspectivas.

\section{Método Numérico}

El método de Dinámica de Partículas (PD por sus siglas en ingles) captura el comportamiento mecánico macroscópico de un sistema particulado mediante el cálculo de la trayectoria de cada una de las partículas dentro del sistema. La evolución en el tiempo de dichas trayectorias, las cuales se obtienen mediante la solución explicita de las ecuaciones de movimiento de Newton, determina entonces el flujo global del material granular. En este método, las fuerzas son 
típicamente fuerzas de contacto normales (Hertzianas) repulsivas y fricción tangencial de acuerdo con las derivaciones de Mindlin. Otras fuerzas tales como adhesión o van der Waals pueden incorporarse de manera relativamente simple. En este trabajo se consideran solamente la fuerza normal y tangencial y se consideran partículas elasto-plásticas perfectamente esféricas y sin rugosidad.

El método se basa en el mecanismo más básico de los fenómenos constitutivos en un ensamblaje granular, esto es, las interacciones partícula-partícula en puntos de contacto. Para determinar el movimiento translacional y rotacional de las partículas en el sistema se utiliza la mecánica Newtoniana clásica. Las ecuaciones que describen el movimiento de las partículas son:

Movimiento lineal

$$
\mathrm{m}_{\mathrm{i}} \frac{\mathrm{d} \mathbf{V}_{\mathrm{i}}}{\mathrm{dt}}=-\mathrm{m}_{\mathrm{i}} \mathbf{g}+\sum_{j=1} \mathbf{F}_{c}
$$

Movimiento angular

$$
\mathrm{I}_{\mathrm{i}} \frac{\mathrm{d} \boldsymbol{\omega}_{\mathrm{i}}}{\mathrm{dt}}=\sum_{j=1} \mathbf{F}_{t} \times \mathbf{r}
$$

donde $\mathbf{F}_{c}=\mathbf{F}_{n}+\mathbf{F}_{t} . F_{c}$, es la fuerza total de contacto, $F_{n}$ y $F_{t}$ corresponden a la fuerza normal y tangencial, respectivamente. La interacción partícula-partícula se establece permitiendo que las partículas se superpongan en el punto de contacto. La superposición sirve como parámetro en los modelos de contacto usados para determinar la fuerza de contacto resultante $F_{c}$.

La simulación consiste de $\mathrm{N}=5200$ partículas perfectamente esféricas no cohesivas de masa fija y diámetro $d_{p}$, que forman un lecho empacado tridimensional regular, comprimido por una pared de peso conocido. El sistema está restringido por una pared cilíndrica de radio $\mathrm{R}=10 d_{p}$, con tapas planas. Todas las propiedades son tomadas de la literatura y consisten exclusivamente de las propiedades mecánicas de los sólidos utilizados. Una condición inicial típica para las simulaciones se obtiene mediante la distorsión de partículas localizadas obre una rejilla tridimensional y permitiendo que las partículas se sedimenten bajo la acción de la gravedad. Las partículas se comprimen sobre una pared plana bajo la acción de la pared superior a la cual se le impone un peso especificado. La Figura 2 ilustra los prototipos de lechos empacados utilizados en las simulaciones. 


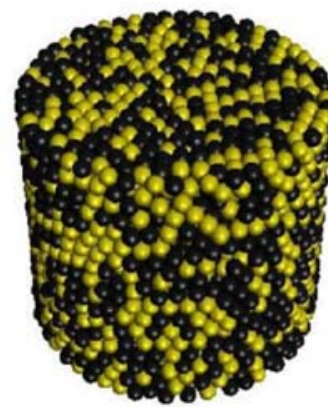

a)

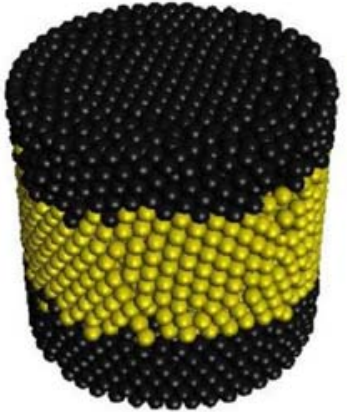

b)

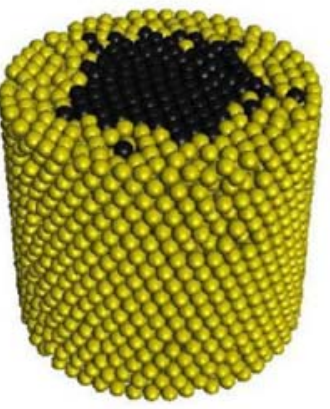

c)

Figura 2. Prototipo de mezclas usadas en la simulación y en los experimentos físicos. (a) Mezclas aleatorias. Mezclas estructuradas, (b) en capas, (c) en núcleos concéntricos. Las esferas negras indican un material duro (acero), las esferas amarillas indican un material blando (polipropileno en este estudio).

\section{RESULTADOS Y DISCUSIÓN}

La validez de las predicciones de los diferentes modelos propuestos en la literatura para la evaluación de la función de distribución de probabilidad $P(f)$, así como los resultados del método numérico de la Sección II pueden evaluarse fácilmente mediante el uso de la denominada técnica del papel carbón desarrollada por los autores de la Ref. [4]. La Figura 3, muestra un esquema del montaje experimental para evaluar la distribución de esfuerzos en un medio granular.
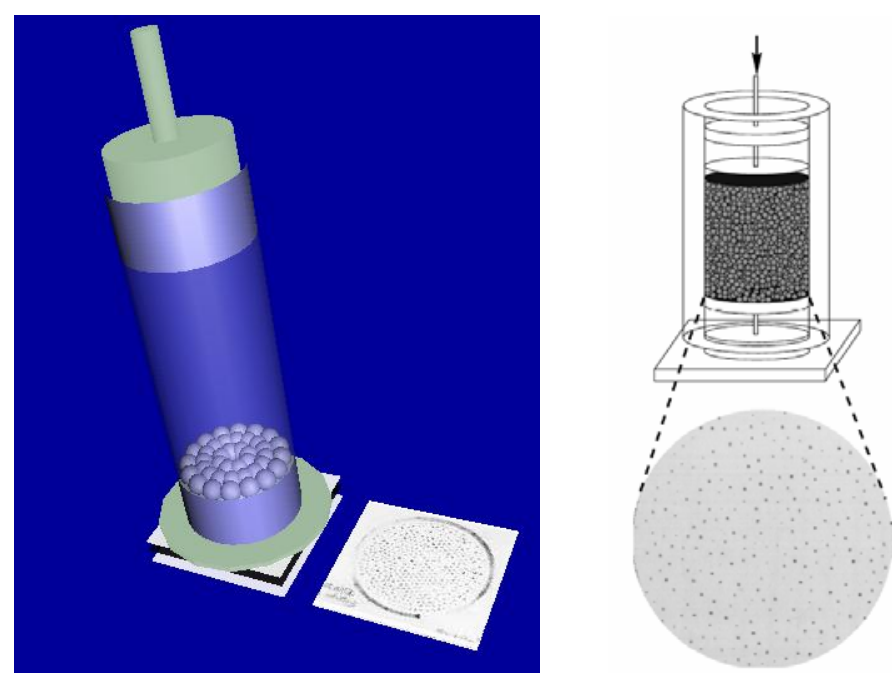

Figura 3. Esquema del aparato utilizado para la toma de datos experimentales. Una carga se aplica al pistón superior y las partículas presionan el papel carbón con el papel blanco, dejando marcas que se utilizan para determinar la fuerza de 
contacto. Un detalle de las marcas sin procesar se muestra en las imágenes adjuntas.

Los lechos tridimensionales de partículas estudiados son lechos compuestos de esferas de acero inoxidable de diámetro $d_{p}=4.76 \pm 0.02 \mathrm{~mm}$ y pellets poliméricos (Polipropileno homopolímero, dureza Rockwell 96) con un diámetro efectivo de $d_{p}=3.17 \pm 0.02 \mathrm{~mm}$, respectivamente. Las partículas se confinan en un cilindro de acero de $150 \mathrm{~mm}$ de altura por $75 \mathrm{~mm}$ de diámetro interno. Una vez el cilindro se llena con las partículas, una carga especificada se aplica al pistón superior usando una prensa hidráulica, mientras se mantiene la pared inferior estática. Las fuerzas de contacto se determinan cubriendo el fondo del cilindro con papel carbón y con papel blanco bajo este. Las partículas en el fondo del cilindro presionan el papel carbón sobre el papel blanco dejando marcas cuyo tamaño e intensidad depende de manera no lineal con la fuerza aplicada sobre la partícula. Una vez culminado el experimento el patrón de marcas se digitaliza usando un scanner convencional y se aplican técnicas de procesamiento de imágenes para extraer la información de interés. Todas las fuerzas para cualquiera de los experimentos se normalizan con la fuerza promedio usando la fuerza promedio para dicho experimento y la distribución de probabilidad es el resultado de promediar tres corridas experimentales independientes. La Figura 4 muestra patrones de marcas típicos obtenidos en el dispositivo experimental de la Figura 3. Puede observarse como el patrón cambia y se hace más cristalino a medida que el porcentaje de material blando en la mezcla se incrementa desde 0 hasta el $100 \%$ de material en el sistema.

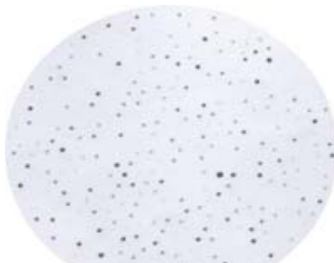

(a)

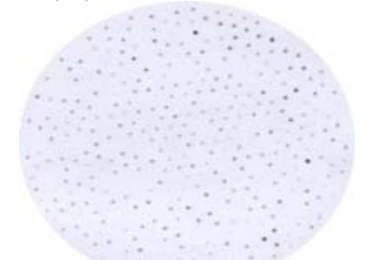

(c)

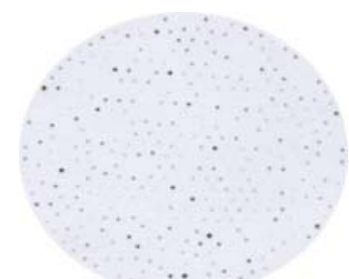

(b)

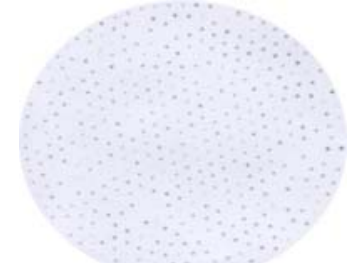

(d)

Figura 4. Patrón de marcas hechas por las partículas sobre el papel blanco localizado en el fondo del cilindro de la Figura 3 para (a) $100 \%$ acero, (b) $20 \%$ 
polímero - $80 \%$ acero, (c) $70 \%$ polímero-30\% acero y (d) $100 \%$ polímero a una carga $F_{n}=20 k N$.

\section{A. Análisis de imágenes}

Los patrones digitalizados de las marcas dejadas por el papel carbón se analizaron utilizando las herramientas de análisis de imágenes desarrolladas por los autores de la Ref.[8]. La herramienta de análisis morfológico pymorph para PYTHON es una colección de herramientas para el análisis de imágenes de código abierto escritas bajo python. El código al igual que algunas demostraciones básicas de su aplicación pueden obtenerse de la página web http://www.mmorph.com/pymorph ó del repositorio de código abierto http://sourceforge.net/projects/pymorph. Comercialmente esta herramienta se puede obtener como un "Toolbox" para MatLab bajo el nombre de Morfología SDC. La implementación de segmentación morfológica se utilizo con el propósito de efectuar la identificación inequívoca de las áreas impresas por partículas individuales. Esta subdivisión que aplica técnicas de segmentación tipo Voronoi, se efectúa de tal forma que la unión de todas las sub-regiones cubra toda la región de interés de la imagen bajo análisis. A menos que se indique lo contrario, todos los análisis se efectuaron sobre imágenes binarias, esto es imágenes blanco y negro donde el color negro se utilizo como fondo. Las coordenadas de las áreas (centroides), áreas y sus distribuciones se determinaron usando el mismo software.

\section{Compresión de una partícula simple}

Para que la técnica del papel carbón pueda ser utilizada con propósitos cuantitativos es necesario efectuar una calibración que establezca la relación entre el área de las marcas impresas por las partículas y la fuerza aplicada. Para tal fin se efectúa la compresión de una partícula individual a diversos niveles de carga de donde se establece una función entre la carga aplicada y alguna característica de la marca dejada por la partícula sobre un papel blanco. La Figura 5 (a) muestra un patrón de calibración previo al proceso de procesamiento, el panel (b) de la Figura 5 muestra el mismo patrón en formato de imagen binaria, tal y como se introduce al software para el análisis de imagen.

\section{(a)}

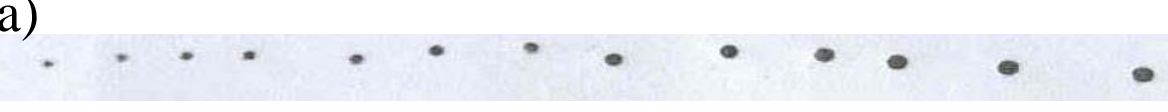

(b)

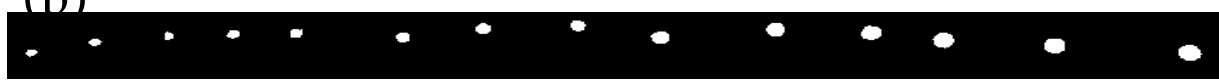


Figura 5. Patrón de marcas hechas por la compresión de una partícula simple como función de la carga. (a) Imagen RGB cruda, (b) Imagen binaria.

Las imágenes en la Figura 6 muestran la identificación de las áreas, la localización de centroides, la numeración de áreas y la obtención de las áreas integradas un proceso que se lleva a cabo de manera totalmente automática.

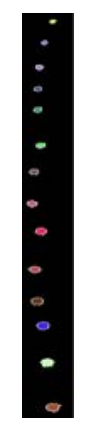

(a)

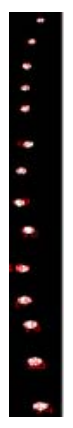

(b)

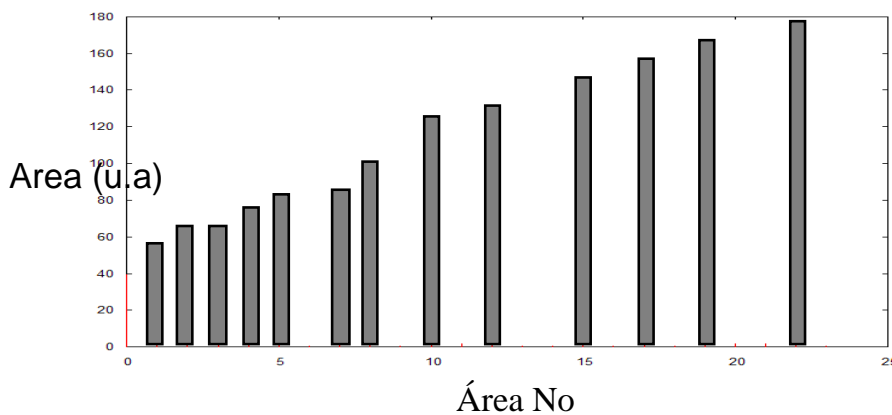

(c)

Figura 6. Procedimiento típico de análisis de imagen para la calibración de la compresión de una partícula simple. (a) identificación de áreas, (b) determinación de centroides e identificación de áreas, (c) integración de áreas.

El procedimiento descrito arriba permite entonces obtener una curva de calibración como la ilustrada en la Figura 7 , la cual muestra la funcionalidad del área impresa como función de la carga aplicada. Dicha curva es la utilizada para efectuar los estudios posteriores sobre empaques de partículas. Los datos en la curva de la Figura 7 representan el promedio de cinco experimentos independientes. 


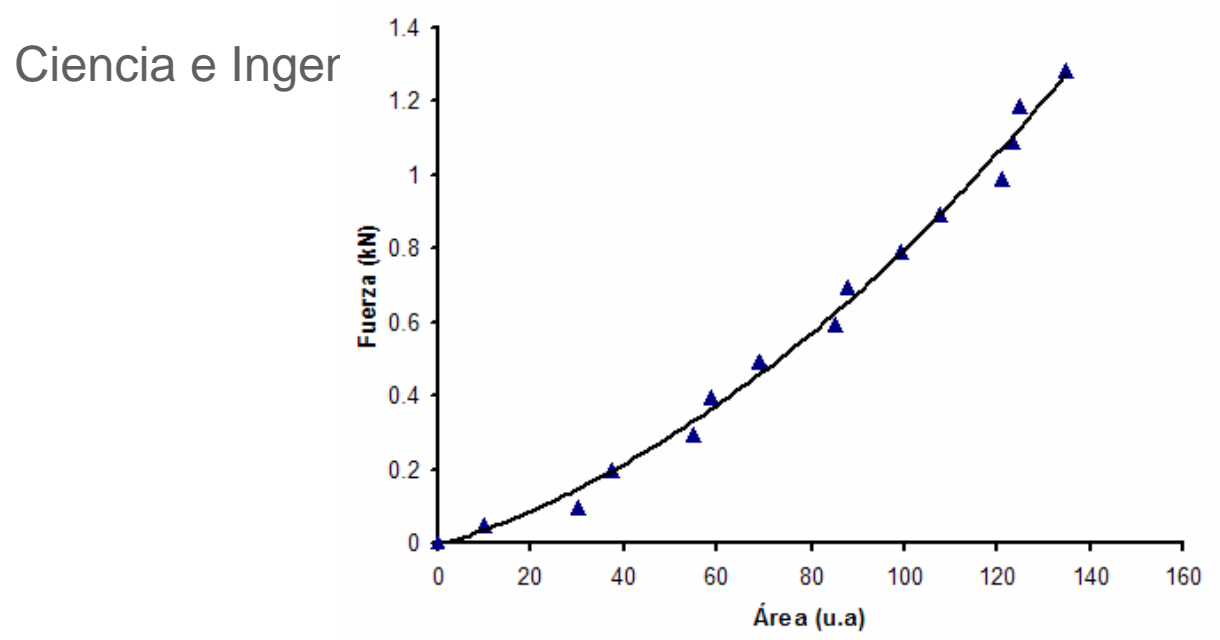

$138-152$

Figura 7. Correlación entre la fuerza experimentada por una partícula simple y el área integrada de los patrones impresos. La línea sólida representa el ajuste de un polinomio de segundo orden.

\section{Compresión de empaques tridimensionales de partículas}

Una vez se ha efectuado la calibración de una partícula simple, se considera entonces la compresión de empaques tridimensionales de partículas. La Figura 4 muestra algunos patrones típicos de marcas crudas en formato RGB sin procesar. Puede observarse claramente como la intensidad de las marcas cambia como función de la composición de la mezcla a un valor de carga constante. Las imágenes de la Figura 4 muestran claramente como el patrón de marcas se hace más uniforme cuando el material se hace más blando, esto es cuando vamos de (a) a (d).

Cambios más evidentes pueden observase cuando se varia la carga manteniendo la composición del sistema. Resultados similares pueden obtenerse también cuando el papel se coloca en la cara superior del cilindro o cubriendo la superficie total del cilindro. Sin embargo, el movimiento lateral de las partículas introduce errores significativos debido a la no circularidad de las marcas. Por esta razón aquí se considera exclusivamente la distribución de fuerzas normales que se ejercen en la cara inferior del cilindro. El procedimiento seguido para el análisis es exactamente equivalente al descrito para la calibración de una partícula simple. La Figura 8 muestra una imagen procesada que incluye la segregación de áreas, su identificación y la localización de centroides. 


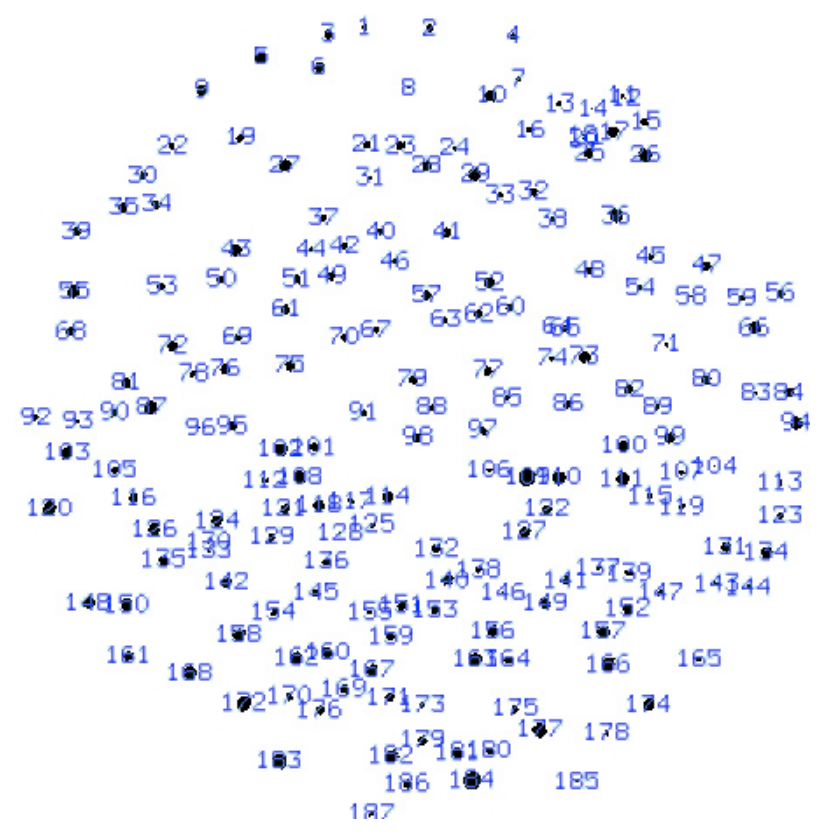

Figura 8. Análisis típico de un patrón de marcas aplicando pymorph para python.

La Figura 9 muestra la evolución de la distribución de probabilidad de fuerzas normales de contacto, durante una prueba de compresión seudo-estática, obtenida mediante la técnica del papel carbón descrita anteriormente.
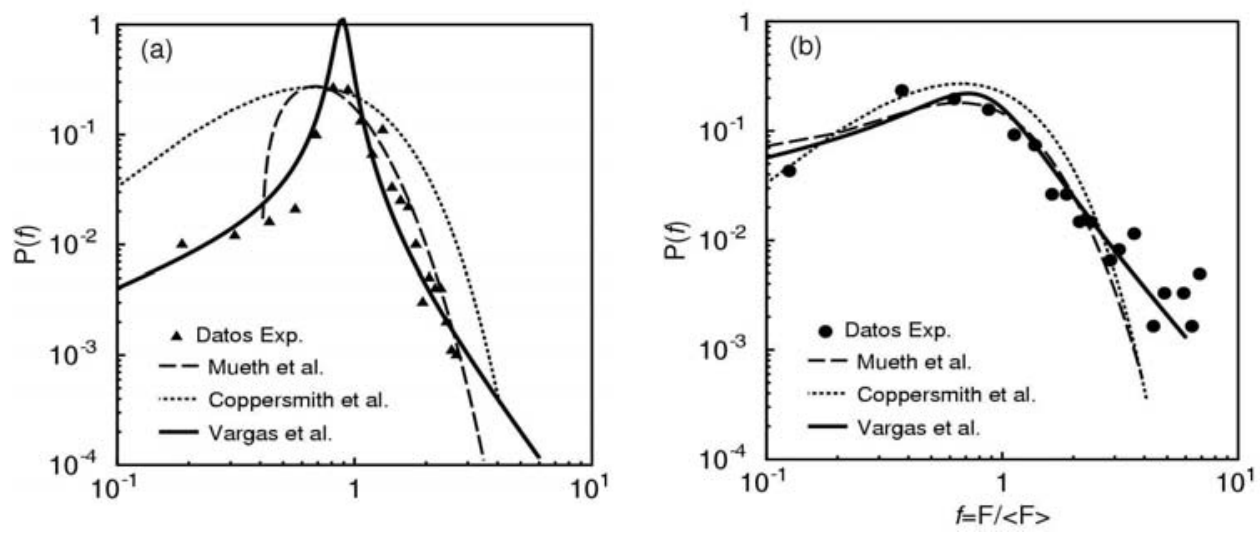

Figura 9. Función de distribución de probabilidad $P(f)$ obtenida aplicando la técnica del papel carbón. (a) partículas poliméricas, (b) partículas de acero para una carga $F_{n}=800 \mathrm{~N}$. Adaptado de Ref.[6].

La Figura 9 compara la distribución de probabilidad obtenida a partir de los experimentos con las predicciones de diversos modelos existentes en la literatura. Tres modelos se comparan en la Figura 9. El denominado modelo $q$ propuesto por Coppersmith et al. [7] 


$$
P(f)=\frac{k^{k}}{(k-1) !} f^{k-1} \exp (-k f)
$$

donde $f=F /<F>$ es la fuerza de contacto normalizada y $k$ es una constante que sirve como parámetro de ajuste. El segundo modelo corresponde a la correlación empírica propuesta por Mueth et al. [4]

$$
P(f)=a\left(1-b e^{-c f^{2}}\right) e^{d f}
$$

donde $a, b, c$ y $d$ son parámetros de ajuste. El tercer modelo corresponde a la correlación semi-empírica recientemente propuesta por los autores de la referencia [6], la cual está dada por

$$
P(f)=z^{-\gamma} K\left(f / z^{\gamma}\right)
$$

donde $\gamma=\beta / \alpha$ es un parámetro de ajuste, $f / z^{\gamma}$ es una variable de similaridad ${ }^{4}$ y la función $K\left(f / z^{\gamma}\right)$ es una función de Wright. Para detalles de su derivación y una comparación más detallada ver la Ref. [6]. En la Figura 9(a), la línea punteada corresponde al ajuste con la Ecuación 3 , con $k=3$. La línea segmentada corresponde a la Ecuación 4, con $a=4.0, b=1.5, c=2.5$ y $d=3.0$. En la Figura 9(b), la línea punteada es semejante al panel (a). La línea segmentada corresponde a la Ecuación 4 , con $a=1.4, b=0.95, c=1.4$ y $d=2.0$. Las líneas continuas son ajustes con el modelo de la Ecuación 5 con (a) $\alpha=\beta=1.45$ (b) $\alpha=\beta=1.9$. Tanto para partículas blandas como duras los datos en la Figura 9 indican que la $P(f)$ obtenida experimentalmente es cualitativamente similar a estudios previos.

En la Figura 10, se compara la distribución de probabilidad obtenida numéricamente bajo condiciones similares a las aplicadas en el experimento físico aplicando la técnica numérica de la Sección II.
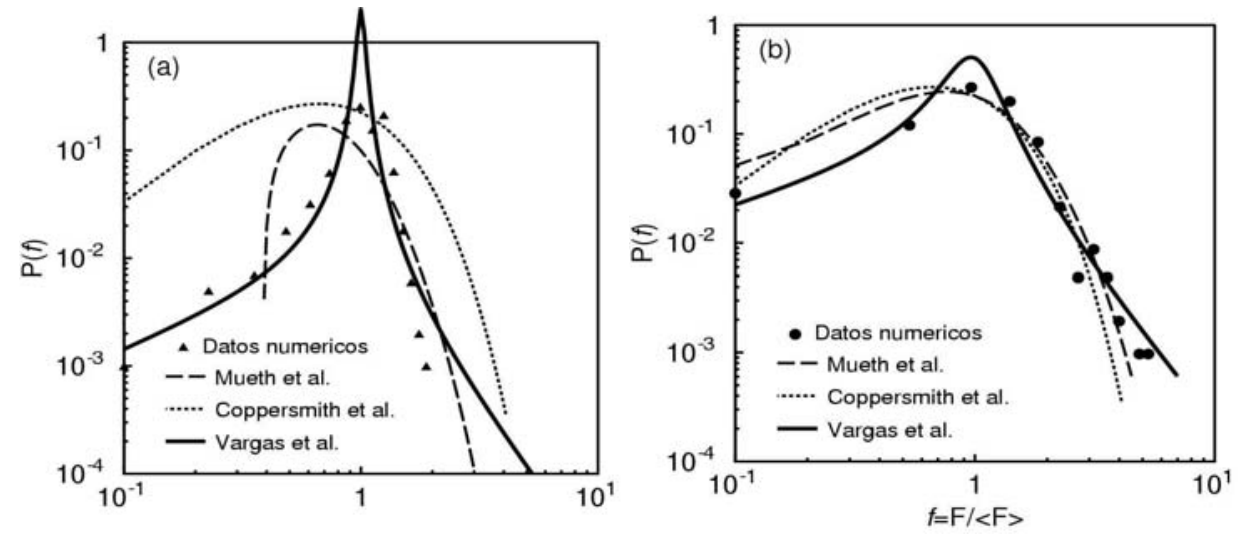

Figura 10. Distribución de probabilidad calculada utilizando el método numérico de la Sección II. (a) partículas poliméricas, (b) partículas de acero para una carga $F_{n}=800$ N. Adaptado de Ref.[6]. 
Notar que el comportamiento observado numéricamente en la Figura 10 es sorprendentemente semejante al obtenido experimentalmente (ver Figura 9). Similar a la Figura 9, la línea punteada representa las predicciones basadas en el modelo teórico en la Ecuación 3 con $k=3$. La línea segmentada corresponde a la Ecuación 4, con $a=4.0, b=1.35, c=2.0$ y $d=3.5$. En la Figura 9(b), la línea punteada es semejante al panel (a). La línea segmentada corresponde a la Ecuación 4, con $a=2.0, b=0.98, c=1.1$ y $d=1.8$. Las líneas continuas son ajustes con el modelo de la Ecuación 5 con (a) $\alpha=\beta=1.95$ (b) $\alpha=\beta=1.7$.

Los resultados en las Figuras 9 y 10 indican que el modelo $q$ ajusta muy pobremente tanto los resultados numéricos como los experimentales. Solo para los sistemas con un bajo grado de deformación (Figuras 9b y 10b) dicho modelo captura apropiadamente el comportamiento para fuerzas arriba del promedio. El modelo empírico propuesto por Mueth et al. [4] al igual que el denominado modelo $q$ ajusta de manera marginal las observaciones numéricas y experimentales. Las observaciones sugieren que al igual que el modelo teórico de la Ecuación 3 la correlación empírica de la Ecuación 4 funciona apropiadamente tan solo para sistemas con bajos grados de deformación. El modelo propuesto por Vargas et al. [6] si bien describe el comportamiento completo presenta algunas desviaciones especialmente para los sistemas con alto grado de deformación (Fig. 9(a) y 10(a)). La Figura 11 muestra como la distribución de probabilidad evoluciona durante una prueba de compresión como función de la composición de la mezcla aleatoria $(\mathrm{S}=\%$ de material blando, $\mathrm{H}=$ $\%$ de material duro) a un valor de carga constante de $25 \mathrm{kN}$. 

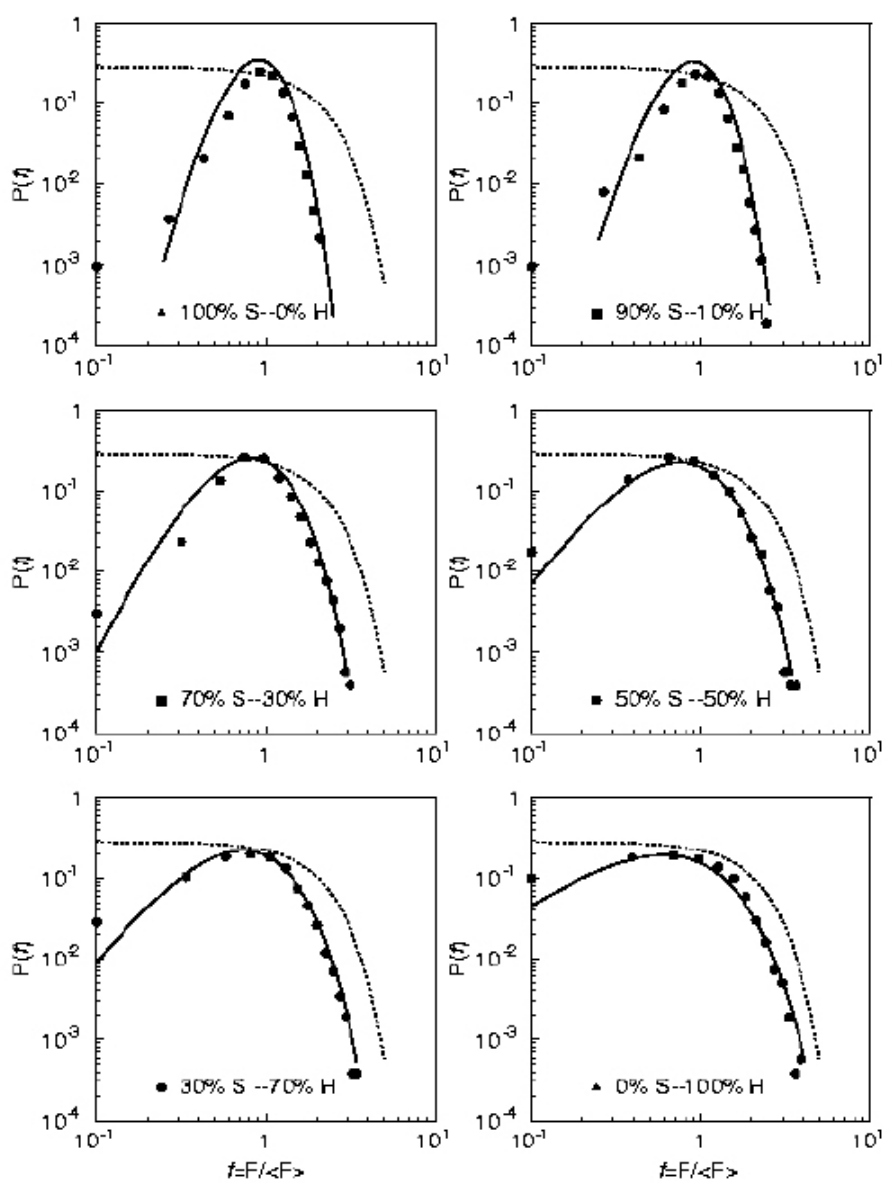

Figura 11. Distribución de probabilidad de las fuerzas normales de contacto como función de la composición de la mezcla. Los datos corresponden a una carga de 25 kN. La línea punteada representa una distribución Gaussiana. La línea continua es una guía para la vista.

Los datos en la Figura 11 indican que en todos los casos la distribución $P(f)$ se desvía del comportamiento Gaussiano clásico, aquí representado por las líneas punteadas. Esta desviación es más pronunciada a medida que se incrementa el porcentaje de material blando $(\% \mathrm{~S})$ en el sistema. En el rango de mezclas considerado los datos indican que la distribución de fuerzas es altamente dependiente del grado de deformación de las partículas.

\section{CONCLUSIONS Y PERSPECTIVAS}

En este artículo, se han presentado algunos resultados experimentales y computacionales sobre la compresión de sistemas mono-dispersos y mezclas binarias de partículas con diferentes niveles de dureza. Se ha mostrado que para sistemas mono-dispersos a un nivel de carga fijo la distribución de probabilidad de las fuerzas de contacto es altamente dependiente del grado de dureza del material. Los resultados de compresión de mezclas binarias de material duro y blando muestran que la distribución es altamente dependiente de 
la composición de la mezcla. Resultados (no mostrados) sobre las características estructurales internas (función radial de distribución y función de correlación) de las mezclas muestran que dichos parámetros no dependen significativamente de la composición. La micro-estructura de las mezclas determinado mediante la distribución de normales de contacto (una medida indirecta de la anisotropía) indican una dependencia fuerte de la composición.

Desde una perspectiva práctica, las observaciones en este estudio muestran claramente como la adición de un material blando a un empaque de partículas duras modifica la distribución de esfuerzos al igual que la micro-estructura. Esta modificación simple de la micro-estructura tiene consecuencias muy importantes de carácter práctico, tales como el control de las variables internas de una mezcla granular incluyendo la distribución de fuerzas y la anisotropía mediante la manipulación cuidadosa de la composición de la mezcla y la localización espacial de sus componentes. Muchas preguntas aún permanecen abiertas. Trabajos futuros intentarán explorar la relación de la geometría del recipiente contenedor, la geometría de las partículas, el efecto de diferentes protocolos de carga y algunas otras distribuciones espaciales.

\section{Agradecimientos}

Los autores expresan su agradecimiento al personal de los laboratorios de Ingeniería Civil de la Universidad Militar Nueva Granada (UMNG) por su colaboración en el desarrollo de las medidas experimentales. Algunos de los resultados en este artículo hacen parte de las memorias de INGENIAR Internacional 2005, realizado en Medellín Colombia (Mayo 4-6 de 2005).

\section{Referencias}

[1] W. L. Vargas and J. J. McCarthy, AIChE Journal 47, 1052 (2001).

[2] X. Jia, C. Caroli, and V. Velicky, Phys. Rev. Lett. 82, 1863 (1999).

[3] E. Falcon, B. Castaing, and M. Creyssels, Eur. Phy. J. B 38, 475 (2004).

[4] D. M. Mueth, H.J. Jaeger, and S. R. Nagel, Phys. Rev. E 57, 3164 (1998).

[5] S. J. Anthony, Phys. Rev. E 63, 011302 (2000).

[6] W. L. Vargas, J. C. Murcia, L.E. Palacio, and D. M. Dominguez, Phys. Rev. E 68, 021302 (2003).

[7] S. N. Coppersmih, C.-h Liu, S. Majumdar, O. Narayan, and T.A. Witten, Phys. Rev. E 53, 4673 (1996).

[8] E. R. Dougherty and R. A. Lotufo, in Hans-on morphological image processing, 1st edition, edited by A. R. Weeks, Jr. (SPIE press, Washington, 2003), Vol. TT59, Chap. 3, p.45. 